\title{
Characterization of a novel endothelial biosensor assay reveals increased cumulative serum inflammatory potential in stabilized coronary artery disease patients
}

Heidi Cung ${ }^{1}$, Mario J Aragon ${ }^{1}$, Katherine Zychowski ${ }^{1}$, Joe R Anderson², James Nawarskas², Carlos Roldan³, Akshay Sood $^{3}$, Clifford Qualls ${ }^{4}$ and Matthew J Campen ${ }^{*}$

\begin{abstract}
Background: Vascular disease is promoted by systemic inflammation that can arise from sites distal to the affected vessels. We sought to characterize the net inflammatory potential of serum from patients with coronary artery disease (CAD) using cultured endothelial cells as a cumulative biosensor.

Methods and results: Serum samples from CAD patients $(N=45)$ and healthy control subjects $(N=48)$ were incubated with primary human coronary artery endothelial cells at a 1:10 dilution for $4 \mathrm{~h}$, followed by isolation of the cellular RNA. Alteration of inflammation-responsive elements (adhesion molecules and cytokines) was assessed by gene expression. Specific indicators included intercellular adhesion molecule-1 (ICAM-1), vascular cell adhesion molecule-1 (VCAM-1), and interleukin-8 (IL-8). Additionally, the cytokine levels in serum samples from all subjects were quantified. Serum from CAD subjects induced greater endothelial ICAM-1, VCAM-1, and IL-8 expression compared to healthy control serum ( $p<0.001$ for each analysis). The three indicators of inflammatory potential (ICAM-1, VCAM-1, and IL-8 mRNA) trended independently of each other and also of serum inflammatory biomarkers. IL-8 expression correlated negatively with serum HDL levels but positively correlated with VLDL, plasminogen activator inhibitor-1 and C-reactive protein. Interestingly, serum levels of cytokines in CAD patients were not statistically different from healthy control subjects. A year of follow-up in a sub-group of CAD subjects revealed relatively stable measures.

Conclusions: As yet unidentified circulating factors in the serum of CAD patients appear to activate endothelial cells, leading to upregulation of adhesion molecules and chemokines. This cumulative assay performed well in terms of discriminating patients with CAD compared to healthy subjects, with greater range and specificity than specific inflammatory markers.
\end{abstract}

Keywords: Coronary artery disease, Biomarker, Inflammatory, Serum, Endothelial

\section{Introduction}

Vascular disease is an inflammatory condition wherein activated endothelial cells mediate the recruitment of immune cells into damaged areas of the vessel wall, promoting oxidative injury, pathological lesion growth and increased histological complexity [1]. Systemic inflammation arising at distal sites, such as the lungs or liver,

\footnotetext{
*Correspondence: mcampen@unm.edu

'Department of Pharmaceutical Sciences, College of Pharmacy, University of New Mexico, Albuquerque, NM, USA

Full list of author information is available at the end of the article
}

may also contribute to the inflamed state of coronary or cerebral vessels through unknown mechanisms [2-6]. Circulating factors likely have a causal role in promoting endothelial cell activation, a state characterized by presentation of adhesion molecules and release of chemokines $[7,8]$. Specific circulating acute phase proteins such as $\mathrm{C}$-Reactive Protein (CRP) and tumor necrosis factor- $\alpha$ $(\mathrm{TNF} \alpha)$ are both associated with vascular disease and can independently activate endothelial cells via NF- $\mathrm{KB}$ pathways [9-11]. However, because the blood contains thousands of factors, the discrete assessment of specific

\section{Ciomed Central}

(C) 2015 Cung et al.; licensee BioMed Central. This is an Open Access article distributed under the terms of the Creative Commons Attribution License (http://creativecommons.org/licenses/by/4.0), which permits unrestricted use, distribution, and reproduction in any medium, provided the original work is properly credited. The Creative Commons Public Domain Dedication waiver (http://creativecommons.org/publicdomain/zero/1.0/) applies to the data made available in this article unless otherwise stated. 
cytokines may fail to capture the overall inflammatory influence that the blood conveys to the endothelial wall. Not surprisingly, such individual factors contribute only modestly to cardiovascular risk prediction or reclassification beyond conventional Framingham risk factors [12] and we propose that this gap between biomarkers and outcome is blurred by the complex composition of the blood and numerous unaccounted factors.

We have developed a novel, less biased approach to assess what we believe to be a functional index of the total inflammatory potential of the serum [13]. By assessing canonical inflammatory response patterns in primary human coronary artery endothelial cells (hCAECs) treated with dilute samples of serum or plasma, the endothelial cells act as biosensors of the complete serum milieu. This assay was initially developed to explore the relatively modest impact of inhaled pollutants on cardiovascular health. It was found that serum from healthy individuals, when obtained shortly following controlled exposures to diesel engine emissions or nitrogen dioxide, carried a greater potential for inducing endothelial cell adhesion molecules and chemokines compared to sham exposure [13]. Similarly, whole serum has been used to identify a net inflammatory influence of cigarette smoking, leading to reductions of eNOS expression and increased reactive oxygen species $[14,15]$. Conversely, in a larger cohort of healthy subjects taking a month-long regimen of a resveratrol-containing nutraceutical, we found that circulating inflammatory potential decreased relative to pretreatment plasma, while placebo administration caused no clear trend and no changes in other biometrics were observed [16]. The overall approach has also proven useful in animal toxicology research $[17,18]$, but the linkage to clinically-relevant disease has yet to be assessed.

Thus, the value of this assay paradigm remains uncertain with respect to clinical outcomes. Many questions remain unanswered, such as whether elevations in net serum inflammatory potential contribute to chronic cardiovascular disease and whether such measures have predictive or diagnostic value. The present study conducted a side-by-side comparison of stabilized coronary artery disease patients on standard-of-care medication with a separate cohort of healthy subjects.

\section{Methods}

\section{Patient population}

Patients with CAD were recruited while hospitalized at the University of New Mexico Hospital for an acute coronary syndrome event (unstable angina, non-ST elevation myocardial infarction, or ST-elevation myocardial infarction) to participate in a health outcomes study designed to assess the benefit of an interdisciplinary cardiovascular risk reduction clinic (CRRC), as compared to usual care. The study was approved by the University of
New Mexico Health Sciences Center Human Research Review Committee and all study patients provided informed consent.

As part of the study, blood samples were collected from all patients for assessment of a variety of traditional and non-traditional risk factors (lipids, HbA1c, homocysteine, malondialdehyde [MDA], plasminogen activator inhibitor-1) and a portion of the samples were stored for future use. Patients randomized to the CRRC were evaluated by both a cardiologist and a clinical pharmacist, who devised an appropriate treatment plan based upon identified cardiovascular disease risk factors. Patients randomized to usual care received followup at the discretion of their primary care providers and/ or specialist providers.

The majority of CAD patients were on standard-of-care pharmacotherapy, including strategies to reduce cholesterol, glucose, blood pressure, platelet aggregation, and tobacco cessation where appropriate. The serum samples used in this analysis were collected at the initial outpatient follow-up study visit to the CRRC.

Healthy control subjects were recruited through the University of New Mexico Clinical and Translational Science Center for routine health screens. Subjects were recruited by newspaper or radio advertisements from the community. Exclusion criteria included - 1) history of diabetes mellitus, atherosclerotic cardiovascular disease, chronic kidney disease, or anorexia nervosa; 2 ) use of statin class of drugs; 3 ) current smoking or having quit smoking within previous two months; 4) pregnancy and nursing state; 5) presence of lung diseases other than asthma; 6) stroke in prior 3 months; 7) aortic aneurysm; and 8) failure to expectorate adequate-quality sputum in response to induction. In addition, all tests were delayed in the event of an acute infection or surgery within the prior 4 weeks and respiratory tract infections or asthma exacerbations within the prior 8 weeks to minimize the effect of these factors on measurements. For actively menstruating women, the testing was done within 3-14 days following the cessation of menstrual flow (a period of high estrogen and low progesterone) to standardize the effect of sex hormones.

\section{Bioelectrical impedance}

CAD participants were assessed for body composition in a fasting state using a Quantum II Bioelectrical Impedance Analyzer (RJL Systems, Clinton Township, MI) using the method described by Heyward [19]. Results for bioelectric impedance were analyzed with Comprehensive Body Composition Software (Human Kinetics, 1997, Champaign, Ill) and percent body fat mass was calculated using standard reference equations appropriate for race and gender [19]. 


\section{Cell culture assays}

The endothelial cell biosensor assay (Figure 1) was conducted as previously described $[13,16]$. Briefly, hCAECs (Lonza, Allendale, NJ) were seeded in a 24-well plate and grown to confluence in complete media (Lonza). The cells were then serum-starved with Basal Media (Lonza) for 24 hours prior to exposure. Confluent hCAEC were incubated with $10 \%$ serum obtained from coronary artery disease (CAD) patients (ie., $50 \mu \mathrm{l}$ serum in $450 \mu \mathrm{l}$ media, per well) or $10 \%$ serum obtained from otherwise healthy individuals. Each plate of cells was treated with approximately equal numbers of subjects from each group in a randomized, blinded fashion. The samples were incubated for 4 hours at $37^{\circ} \mathrm{C}$. RNA was extracted from the cells and cDNA was made using a ThermoCycler (Model \# PTC200; MJ Research). The cDNA was used to determine gene expression via quantitative polymerase chain reaction (LightCycler 480 II, Roche, Indianapolis, IN). Specific targets included interleukin-8 (IL-8), intercellular adhesion molecule-1 (ICAM-1), and vascular cell adhesion molecule-1 (VCAM-1), with TATA-box protein used as a housekeeping gene.

\section{Serum cytokine measurements}

Serum samples from healthy and CAD patients were analyzed using an electrochemiluminescence detection system (Meso Scale Discovery, Rockville, MD). The serum samples were analyzed for the presence of CRP, soluble ICAM-1, soluble VCAM-1, serum amyloid A (SAA), TNF- $\alpha$, IL-6, IL-8, and IL-1 $\beta$ using commercially-available kits.

\section{Data analysis}

Gene expression data were $\log _{10}$-transformed for normality. Data were analyzed between groups (CAD versus control) via Satterthwaite's t-test (SAS v9.4). A multivariate analysis confirmed this difference between CAD and control outcomes after adjusting for age, sex, and BMI. Correlational (Spearman) and multivariate analysis was carried out on the CAD patient outcomes relative to demographic and clinical data (SAS).

\section{Results}

\section{Cohort data}

A total of 48 patients with CAD and 45 healthy control subjects were included in the study. Subjects were selfselected and inclusion criteria allowed for a wide range of CAD etiology and manifestations. Table 1 summarizes the demographic information. The CAD patients in this unmatched study were expectedly older than control subjects and had a disproportionate prevalence of $\mathrm{CV}$ risk factors, including diabetes, body mass index (BMI), hypertension, hypercholesterolemia and current tobacco use. Importantly, a sufficiently overlapping range of demographic factors permitted multivariate assessment to eliminate all potential major effect modifiers, in terms of endothelial cell responses to serum, described below. The CAD cohort was comprised of patients with single and multiple vessel disease, and were recorded on admission as being a mix of non-ST segment elevation myocardial infarction (NSTEMI) and ST segment elevation myocardial infarction (STEMI), as well as unstable angina. Table 2 provides all additional personal health data from the CAD patient population that were available.

\section{Serum from CAD patients has greater inflammatory potential than serum from healthy subjects}

hCAECs treated with $10 \%$ serum from CAD subjects showed substantial increases in IL-8, ICAM-1, and VCAM-1 expression, compared to hCAECs treated with serum from healthy control subjects (Figure 2). Endothelial mRNA expression of IL-8 and VCAM-1 were approximately $80 \%$ higher in hCAECs treated with CAD patient serum compared to controls $(\mathrm{p}<0.0001)$. ICAM-1 expression was more stable at approximately $30 \%$ above control $(P=0.0009)$ and with less overall variability. The distribution patterns of gene expression for each of these

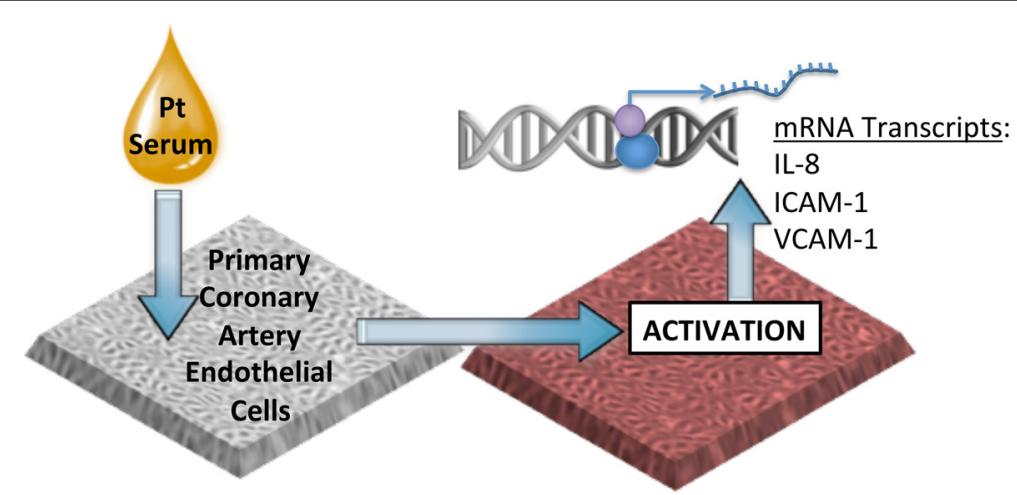

Figure 1 Endothelial cell biosensor approach. Primary coronary artery endothelial cells are incubated with 10\% serum from individual patients for $4 \mathrm{~h}$, followed by isolation of RNA, and then assayed for transcriptional responses. 
Table 1 Basic demographics for the healthy and coronary artery disease subjects

\begin{tabular}{|c|c|c|}
\hline & $\begin{array}{l}\text { Healthy controls } \\
(\mathrm{N}=45)\end{array}$ & $\begin{array}{l}\text { Coronary artery } \\
\text { disease patients } \\
(\mathrm{n}=48)\end{array}$ \\
\hline Age (Mean $\pm S E$; range) & $31.7 \pm 13.5 ; 18-61$ & $56.5 \pm 7.9 ; 36-77$ \\
\hline Gender (\% female) & $71 \%$ & $41.7 \%$ \\
\hline $\mathrm{BMI}$ (Mean $\pm \mathrm{SE}$; range) & $22.3 \pm 1.7 ; 18.7-25.5$ & $29.9 \pm 6.4 ; 17.2-48.2$ \\
\hline $\begin{array}{l}\text { Fat composition } \\
\text { (Bioelectric impedence) }\end{array}$ & $25.27 \pm 6.5 ; 12.0-40.0$ & $34.3 \pm 10.5 ; 18.5-56.3$ \\
\hline \multirow[t]{4}{*}{ Race } & $\begin{array}{l}\text { 9.3\% Native } \\
\text { American/Alaskan }\end{array}$ & 57.9\% Hispanic \\
\hline & 4.7\% Asian & 36.8\% White \\
\hline & 9.3\% African & 5.3\% Other \\
\hline & $\begin{array}{l}76.7 \% \text { Caucasian } \\
\text { (29\% Hispanic) }\end{array}$ & \\
\hline \multicolumn{3}{|l|}{ Comorbidities } \\
\hline Diabetes & $0 \%$ & $58.3 \%$ \\
\hline Hypertension & $0 \%$ & $22.9 \%$ \\
\hline Smoking & $0 \%$ & $50 \%$ \\
\hline $\begin{array}{l}\text { Extent of CAD (single, } \\
\text { multi vessel), } N(\%)\end{array}$ & NA & 20 (42\%), 28 (58\%) \\
\hline $\begin{array}{l}\text { Reason for admission } \\
\text { (NSTEMI, STEMI, unstable } \\
\text { Angina), N (\%) }\end{array}$ & NA & $\begin{array}{l}14(29 \%), 21(44 \%), \\
13(27 \%)\end{array}$ \\
\hline
\end{tabular}

markers were notably different and correlations among the marker expression from individuals varied considerably. Cross-correlations ranged from moderate $(R=0.534$ for VCAM-1 and ICAM- 1 ) to low ( $\mathrm{R}=0.201$ for ICAM- 1 and IL-8), implying that these responses might be influenced by different factors - or cumulative factors - in the serum.

\section{Relationship of endothelial responses to serum to time post-event and longitudinal trends}

As many serum components can be acutely altered as a result of major cardiac events, or may trend with age and/or time, we examined the impact of temporality on serum-treated hCAEC mRNA expression in both the cross-sectional samples, as well as with a sub-cohort of longitudinally followed CAD subjects. All samples were obtained from patients with known CAD, either from having previously suffered an acute myocardial infarction or angina attack. The average time post-event was slightly greater than one year (400 days), but 5 subjects enrolled in the study within 2 months of the precipitating event and 6 subjects were greater than 2 years past their event. The earliest enrolled subject had an event 16 days prior to the initial visit. In general, this crosssectional data set did not reveal strong trends of temporal
Table 2 Further clinical data from CAD cohort

\begin{tabular}{|c|c|c|c|c|}
\hline & Mean & SD & Min & Max \\
\hline Total cholesterol (mg/dl) & 149.9 & 39.4 & 76 & 241 \\
\hline LDL & 88.6 & 31.7 & 34 & 175 \\
\hline HDL & 40.2 & 12.1 & 25 & 88 \\
\hline HDL2 & 9.3 & 4.9 & 4 & 32 \\
\hline HDL3 & 30.9 & 7.9 & 18 & 56 \\
\hline VLDL & 22.0 & 8.3 & 12 & 64 \\
\hline VLDL 1,2 & 9.8 & 3.2 & 5.6 & 20 \\
\hline VLDL 3 & 18.1 & 46.2 & 7 & 341 \\
\hline Triglycerides & 156.1 & 74.8 & 5 & 323 \\
\hline $\operatorname{Lp}(a)$ & 6.8 & 3.9 & 3 & 17 \\
\hline IDL & 8.4 & 5.5 & 1 & 24 \\
\hline Glucose (mg/dL) & 115.8 & 37.5 & 81 & 265 \\
\hline $\mathrm{HbA1c( \% )}$ & 7.63 & 1.88 & 5.4 & 11.4 \\
\hline Homocysteine & 9.25 & 4.00 & 4.6 & 24.6 \\
\hline Insulin (ulU/mL) & 14.09 & 8.02 & 2.4 & 43.5 \\
\hline Fibrinogen (mg/dL) & 363.2 & 89.8 & 231 & 660 \\
\hline PAI (IU/ml) & 15.1 & 13.1 & 0.1 & 50 \\
\hline UrMicAlb (mgALB/gCrea) & 77.1 & 271.6 & 0.6 & 1481.6 \\
\hline SBP & 130 & 19 & 100 & 186 \\
\hline DBP & 74 & 11 & 48 & 100 \\
\hline HR & 67 & 11 & 43 & 89 \\
\hline Weight(kg) & 85.5 & 17.4 & 53.4 & 123.25 \\
\hline Bioelectric impedence & 36.1 & 9.08 & 22.6 & 48.8 \\
\hline MDA & 0.236 & 0.089 & 0.094 & 0.414 \\
\hline Days post-event & 400 & 568 & 16 & 2748 \\
\hline \multicolumn{5}{|l|}{ Medication data } \\
\hline Statin use & $75 \%$ & & & \\
\hline Antiplatelet use & $97.9 \%$ & & & \\
\hline Glucose control & $25 \%$ & & & \\
\hline ACE inhibitors or ARBs & $75 \%$ & & & \\
\hline$\beta$-blockers & $68.8 \%$ & & & \\
\hline
\end{tabular}

resolution of the bioactivity of the serum (Figure 3A-C). For serum-treated hCAEC IL-8 expression, there were no significant trends. hCAEC mRNA for ICAM-1 and VCAM-1 demonstrated modest reductions relative to the time post-event $\left(P=0.033\right.$ and $0.055, R^{2}=0.10$ and 0.08 , respectively). As serum samples more proximal to the event than 16 days were not available, it is not known if the serum obtained within hours or days after the event might be more inflammatory. However, from months-to-years after an event, the data suggest considerable stability.

In a limited cohort of subjects $(\mathrm{N}=11)$, four longitudinally obtained serum samples were available for approximately one year after the initial clinic visit (Figure 3D-F). 


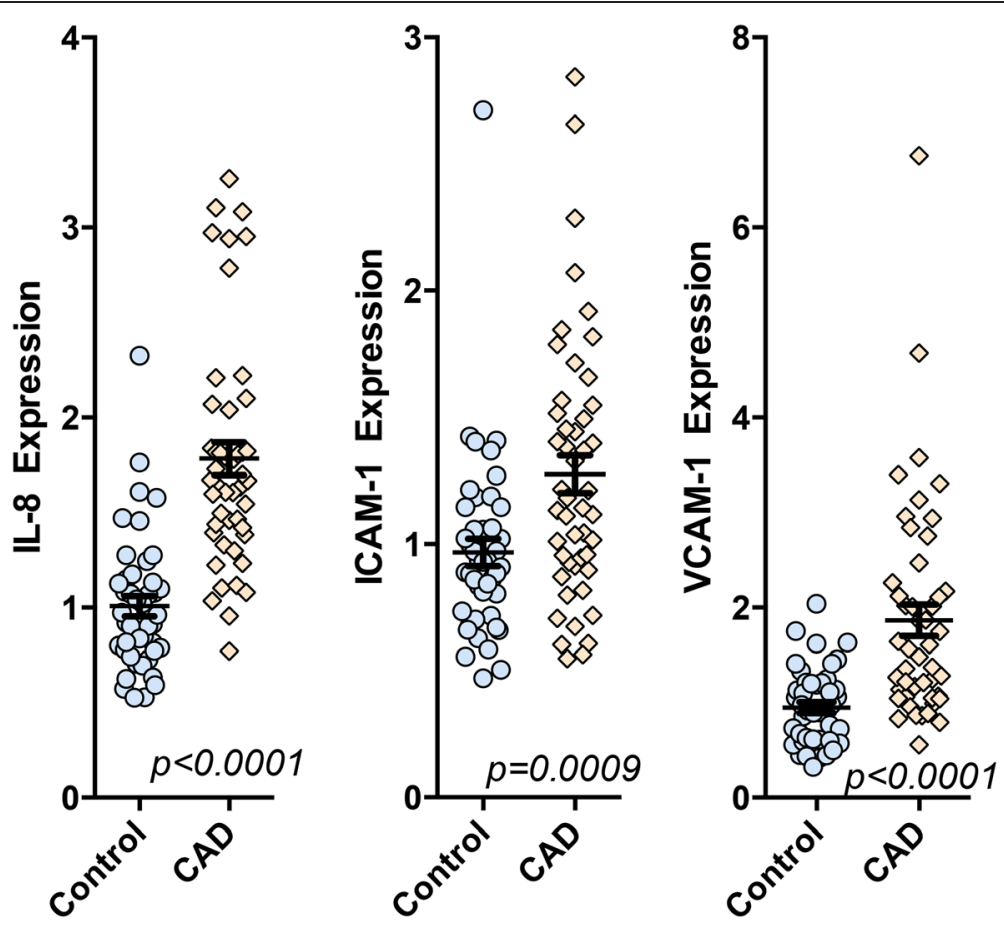

Figure 2 Endothelial cell expression of IL-8, ICAM-1, and VCAM-1 mRNA in response to incubation with $10 \%$ serum from healthy controls or CAD cohort. Significance was assessed with a Student's t-test. The differences between groups remained significant after controlling for age, sex, and body mass index, and all potential interactions using a multivariate analysis of variance (general linear model).

We examined the relative serum inflammatory potential trends, in terms of activation of hCAEC responses, in these subjects and found that no absolute trends were observed over the year of follow-up. However, clear variability in endothelial cell responses to serum obtained at different visits was evident. As the medical records for these subjects lacked temporal details in terms of symptoms and medications, we are unable to ascribe such short-term trends to any clear change in health status. Importantly, the lack of long term trends in this sub-cohort of CAD subjects on standard of care medication is consistent with the fact that all of these subjects survived beyond this year of follow-up.

\section{CAD Manifestation effects on serum inflammatory potential}

Medical records allowed for initial event categorization of CAD subjects. We therefore considered an intra-cohort comparison for subjects whose initial presentation was a myocardial infarction (with or without ST elevation) or unstable angina (Figure 4). A slight trend for increased expression of the gene transcripts was noted for patients admitting with an MI, which was only significant for VCAM-1 $(P=0.0266)$. No significant differences were observed between subjects admitting with STelevation MI or non-ST elevation MI (data not shown).
These findings are consistent with the hypothesis that greater inflammatory potential in the serum may lead to more severe vascular pathology, although the results are associative.

Endothelial cell responses to serum were not impacted by sex, age or BMI

Importantly, the significant differences in endothelial expression of IL-8, ICAM-1, and VCAM-1 in response to patient serum remained significant even after adjusting for the potential major effect modifiers of sex, age, and BMI that were clearly different between cohorts. Direct comparisons across sex, age, and BMI are shown in Figure 5. For each mRNA target, it is clear that CAD subjects were elevated compared to healthy controls, but there was not impact of sex on the endothelial cell responses to serum in either healthy or CAD cohorts (Figure 5A-C). Similarly, age and BMI showed no clear trends across the complete range of either variable (Figure 5D-I).

Multivariate regression was also applied to test the transcriptional outcomes relative to the potential effect modifiers. In considering all variables and potential interactions among sex, age, and BMI as confounders, differences between healthy and CAD subjects for expression of IL8, ICAM-1, and VCAM-1 remained significant. Thus, 


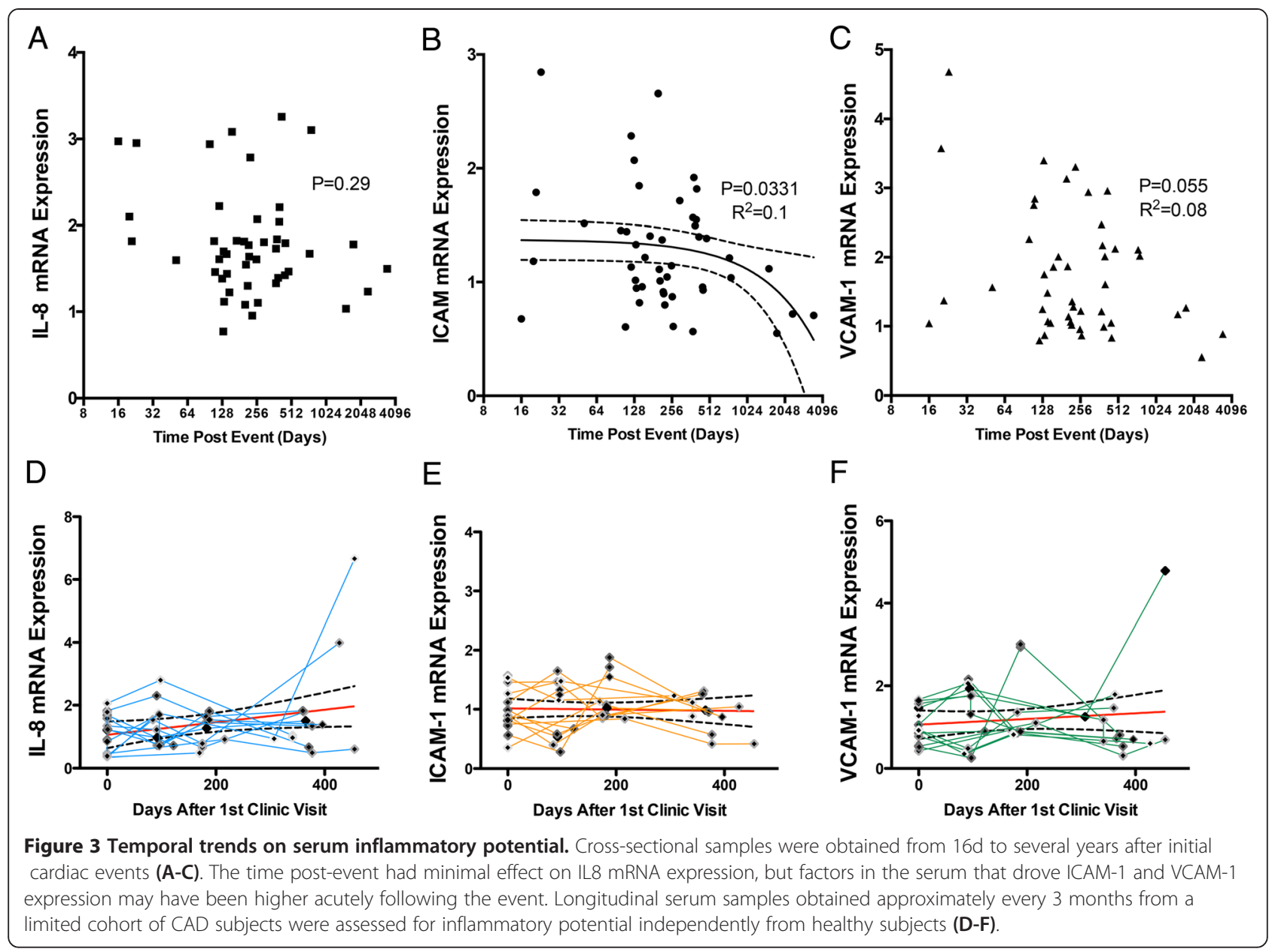

despite the unmatched cohorts, there is high confidence that the CAD condition alone imparts an independent pro-inflammatory effect on the serum.

\section{Demographic factors that correlate with hCAEC responses} In exploring potential factors that may contribute to serum-induced endothelial cell responses, we found a number of demographic factors in CAD patients that correlated with IL-8, ICAM-1 and VCAM-1 expression (Table 3). IL-8 expression associated with a number of factors, positively trending with serum levels of VLDL1/2, CRP, and PAI-1, while negatively trending with serum glucose and HDL levels. VCAM-1 expression correlated with both smoking and serum homocysteine levels, while ICAM-1 expression correlated with age, serum homocysteine, and MDA. While serum homocysteine trended negatively with inflammatory potential, it should be noted that out of 51 patients, only 3 values for homocysteine were out of normal range, and only two of these were paired with a measure for VCAM-1 mRNA. Fewer demographic and personal health data were available for our control population, and those few factors analyzed (age, BMI, gender) did not correlate with endothelial cell responses to serum.

As IL-8 expression had the most robust correlates, we developed a regression model to better understand the factors that predict this outcome (Table 4). This model confirmed the outcomes in the correlational analysis, providing confidence for the roles of serum VLDL, CRP, and glucose levels in driving the IL-8 related inflammatory potential. However, these variables only explained a small portion of the inflammatory potential, indicating that other unmeasured factors are also mediators.

\section{Cytokine Levels did not explain the difference in serum inflammatory potential between groups}

To assess whether inflammatory cytokines might explain differences in the serum inflammatory potential between healthy and CAD subjects, we measured 8 key inflammatory cytokines in serum from all patients using 


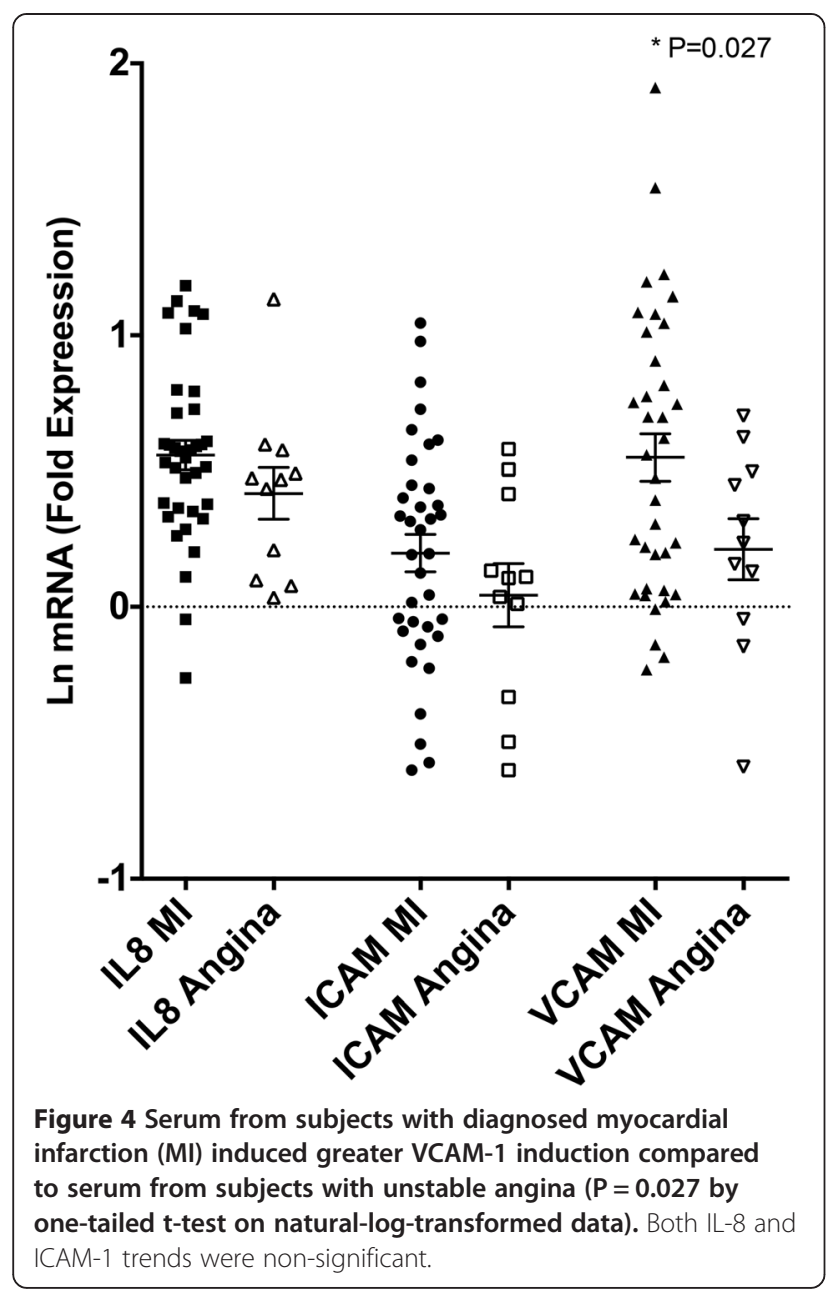

an electrochemiluminescence technique (Figure 6). No statistically significant differences were noted for IL-1 $\beta$, IL-6, IL-8, TNF $\alpha$, C-Reactive Protein, soluble VCAM-1, or Serum Amyloid A, either in terms of mean or distribution, between healthy and CAD cohorts. A modest, but non-significant increase in soluble ICAM-1 was noted however $(49.1 \pm 8.1$ controls versus $84.1 \pm 14.4$ in CAD patients, $\mathrm{P}=0.086$ ).

\section{Discussion}

The present study assessed cumulative serum inflammatory potential from a cohort of CAD patients in stable condition under standard-of-care for medications and lifestyle adjustments, using an endothelial biosensor assay. Utilization of circulating biomarkers has proven valuable for diagnostic and prognostic purposes in atherosclerotic disease, but even the most predictive single mediators provide only small incremental improvements in overall prediction of risk or patient reclassification [20,21].
Endothelial inflammatory response to serum from CAD patients was significantly increased compared to responses to control subject serum, despite observing that most measured circulating cytokines were no different between these cohorts. Additionally, our characterization of the temporal dynamics of this assay reveals stability over the course of a year of follow-up, albeit with some lability, reflecting a generally successful course of treatment. The value of this assay paradigm - using endothelial cells as "biosensors" of circulating inflammatory potential remains incompletely appreciated but highly promising, as the ability to discriminate clinical populations in a manner consistent with a diagnosed clinical condition would be a clear advantage over assessment of single or even multiple biomarkers that can not discriminate from healthy subjects.

Serum obtained from patients with a previous history of a major coronary vascular event showed a greater potential for inducing endothelial cell inflammatory responses, such as IL-8, VCAM-1, and ICAM-1 expression, compared to serum from healthy individuals. The CAD patients, all substantially post-event (mean $=1.1 \mathrm{y}$; range $=16 \mathrm{~d}-7.5 \mathrm{y}$ ), were expectedly receiving numerous medications $(>7)$ as standard-of-care. CAD subjects were generally prescribed at least one lipid control agent (e.g., statin), an antiplatelet agent (e.g., aspirin and/or clopidogrel), and patients with Type 2 Diabetes Mellitus were also taking at least one glucose control agent (Table 2). Many of these medications have reported pleiotropic anti-inflammatory effects, which may explain the reduction of cytokine levels to control cohort values. In recent reports, statins have been shown to reduce CRP levels. For instance 6 months of $10 \mathrm{mg} /$ day of Atorvastatin led to a $26 \%$ drop in CRP and also diminished the predictive value of CRP, in terms of risk for major adverse cardiovascular events [22]. Simvastatin therapy provides similar results, while combined simvastatin and ezetimibe led to a $>35 \%$ reduction on CRP [23]. Other standard of care medications likely contribute further to this CRP lowering effect, including aspirin [24], clopidogrel [25], and glucose control medications such as rosiglitazone [26]. Other inflammatory cytokines, including IL-6 and TNF- $\alpha$, appear to be similarly affected by statins and antiplatelet medications $[27,28]$. Thus, the ability to differentiate healthy control subjects and patients with diagnosed CAD using such cytokine markers is substantially hampered once such medications are implemented [22]. Importantly, serum inflammatory potential determined with the novel endothelial bioassay remained elevated compared to healthy control subjects, despite the medication use, although the prognostic/diagnostic value of such outcomes remains to be defined.

In previous research, we have found this endothelial bioassay paradigm valuable for assessing potential benefits of 


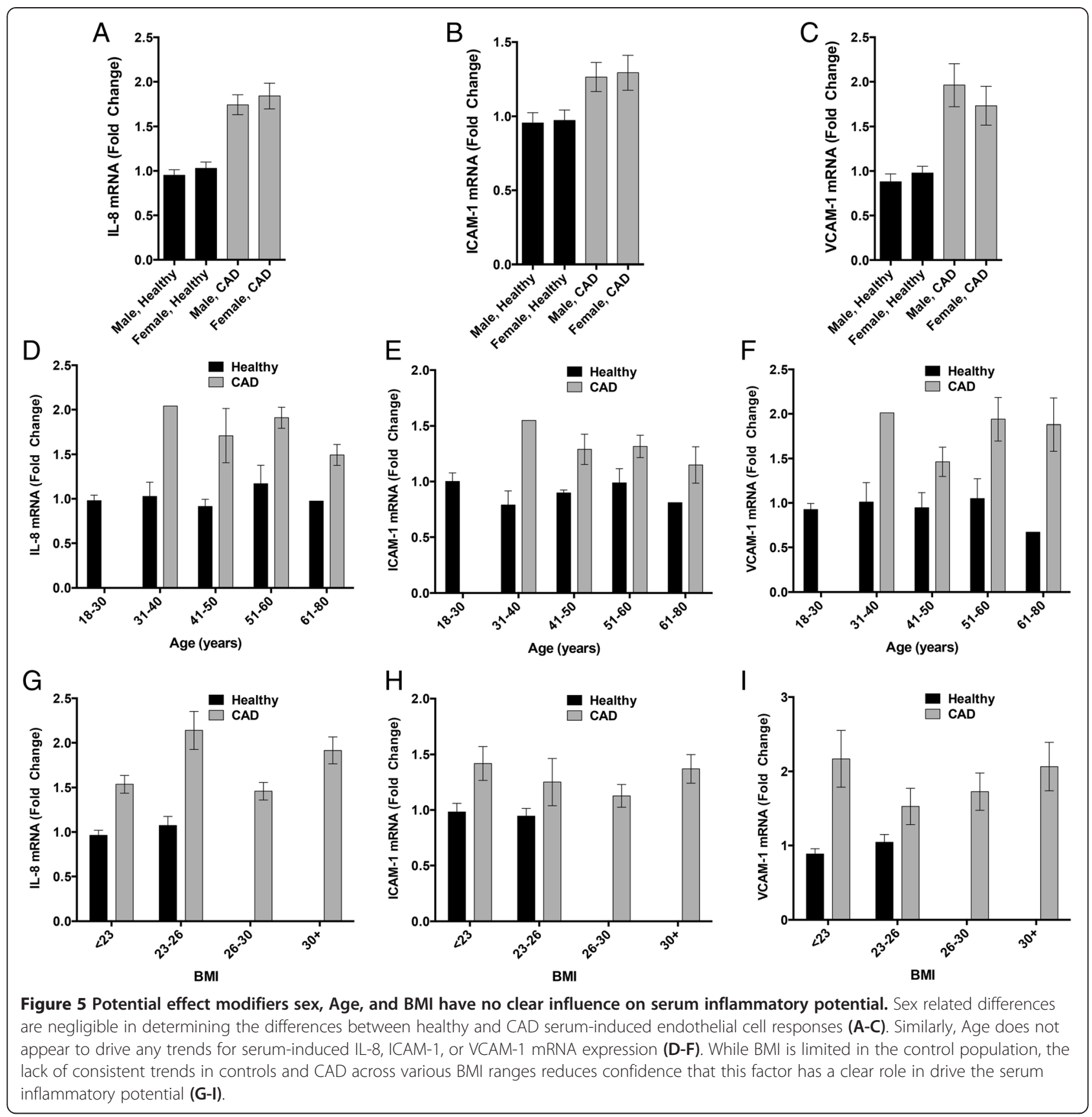

grape seed extract with added resveratrol in a healthy adult population [16]. Whereas serum markers of inflammation, such as interferon-gamma and interleukin-1 $\beta$, did not change with resveratrol/grape seed extract treatment, compared to placebo, endothelial cells incubated with serum obtained after the month-long resveratrol/grape seed extract regimen exhibited consistently lower expression of IL-8, ICAM-1, and VCAM-1 mRNA compared to baseline responses. Conversely, in studies of air pollution health effects in another healthy cohort, we observed an increase in the serum inflammatory potential following inhalation of diesel exhaust emissions and nitrogen dioxide [13]. The degree of inflammatory potential change was smaller than that seen with the differences in the present study, on the order of $20-30 \%$ increases, acutely, compared to $30-90 \%$ increases for mRNA responses to serum between CAD patients and a healthy cohort. These outcomes are consistent with earlier research using this general approach. Barbieri and colleagues found that serum from cigarette smokers could induce COX-2 and 
Table 3 Factors in CAD patients that correlate with hCAECs responses to serum

\begin{tabular}{lll}
\hline IL-8 & $\boldsymbol{R}$ & $\boldsymbol{p}$-value \\
\hline Diabetic & 0.262 & 0.079 \\
Glucose & -0.358 & 0.013 \\
HDL & -0.298 & 0.039 \\
HDL2 & -0.272 & 0.061 \\
HDL3 & -0.283 & 0.052 \\
VLDL1/2 & 0.254 & 0.081 \\
C-Reactive protein & 0.329 & 0.038 \\
Plasminogen activator inhibitor-1 & 0.367 & 0.077 \\
\hline VCAM-1 & $R$ & $p$-value \\
\hline Smoking & 0.263 & 0.074 \\
Homocysteine & -0.274 & 0.059 \\
\hline ICAM-1 & $R$ & $p$-value \\
\hline Age & -0.301 & 0.038 \\
Homocysteine & -0.368 & 0.010 \\
Time post-event & -0.312 & 0.033 \\
\hline
\end{tabular}

intracellular reactive oxygen species in a manner that appeared dependent on TNF $\alpha$ and interleukin-1 $\beta$ [15]. Similarly, Barua and colleagues treated hCAECs with serum from smokers and found a down-regulation of eNOS protein activity that was reversible by the addition of superoxide dismutase and catalase [14]. The present study of CAD provides a vital clinical anchor for understanding these earlier studies that used this serum inflammatory potential assay, showing that elevated endothelial cell responses to serum is consistent with diagnosed vascular disease.

Several study limitations are also viewed as opportunities for future research, as the overall concept of holistic cell-based responses to whole serum remains quite novel and unexplored. For one, while IL-8, VCAM-1, and ICAM-1 mRNA are consistently reported as involved in vascular disease progression, both in clinical studies and basic research, there are a number of important response factors that have not been assessed. It is highly probable that many other transcripts are more responsive to the inflammatory factors in the CAD serum, or that specific proteins may be produced, expressed on the cell surface, or secreted in a selective manner. It is likely that endothelial genomic or proteomic outcome patterns would identify stronger response elements or provide "fingerprint" responses to specific clinical conditions or allow differentiation between acute environmental influences (i.e., diet, air pollution) and chronic syndromes (i.e., type 2 diabetes mellitus or end stage renal disease).

Secondly, we have yet to clearly link endothelial cell responses to serum with outcomes of morbidity and/or mortality, thus the clinical value of the present study remains uncertain. Our previous work suggests that this approach may be valuable for studies of toxicology [13] or therapeutic efficacy [16], and the present study highlights a potential for diagnostic purposes. However, we did not have sufficient subject numbers to conduct a complete follow-up study to determine whether the serum inflammatory potential may help in the prediction of adverse outcomes.

Lastly, there is inherently concern relative to the unmatched study cohorts, despite our findings revealing negligible influence of sex, age, or BMI on the coronary artery endothelial cell transcriptional responses to CAD patient serum. Improved matching in future research will certainly provide greater confidence in this outcome. However, our findings remain notable due to the power of this novel approach to discriminate these populations when conventional inflammatory cytokines did not. There is a vast amount of clinical and research efforts invested in the use of circulating biomarkers. The finding in the present study that CAD patients on standardof-care medication showed no difference in cytokine profiles from a cohort that was younger, had a lower $\mathrm{BMI}$, and more predominantly female, is naturally a concern and clear motivation for the development of novel diagnostic and prognostic markers, such as pursued in the present study.

\section{Conclusions}

The use of cells as biosensors to detect cumulative circulating inflammatory may have significant clinical value. Serum contains thousands of factors - proteins, lipids, and metabolites - that can be augmented by numerous pathologies. Hundreds of potential drivers of endothelial cell activation and vascular inflammatory pathologies exist, such as myeloperoxidase, oxidized lipids or lipoproteins,

Table 4 Predictive variables for IL8 response using a linear regression model

\begin{tabular}{llllll}
\hline Variable & Parameter estimate & Std error & $\mathbf{t}$ value & $\mathbf{P r}>|\mathbf{t}|$ & Standardized estimate \\
\hline Intercept & 0.141 & 0.0815 & 1.73 & 0.092 & 0 \\
VLDL 1/2 & 0.0199 & 0.0062 & 3.22 & 0.0027 & 0.438 \\
CRP & 0.0100 & 0.0028 & 3.54 & 0.0011 & 0.474 \\
Glucose & -0.0012 & 0.0005 & -2.22 & 0.0328 & -0.298 \\
\hline
\end{tabular}




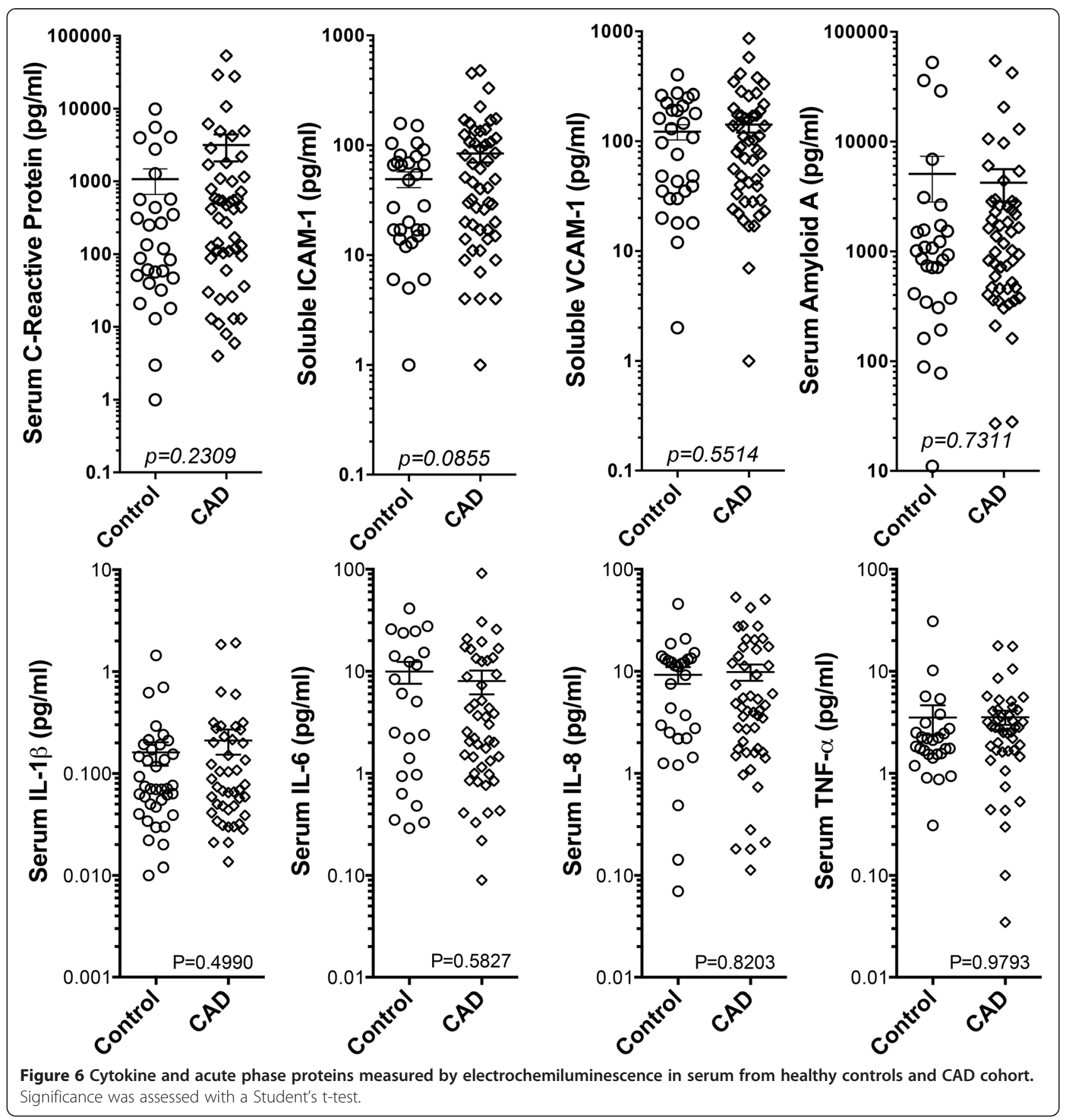

advanced glycation endproducts or damage-associate molecular patterns (DAMPs). Additionally, endogenous proteins may be modified (fragmented or adducted) by reactive molecules, leading to altered biological activity or pathological epitopes. The present findings, combined with previous experience with this assay, highlight the potential usefulness of a cumulative bioactivity response, compared to measurements of individual factors to assess an overall inflammatory potential of the circulation. Sophisticated proteomic, lipidomic and metabolomics approaches will naturally be valuable to establish key drivers and link back to clinical course. However, we remain skeptical that any single marker will explain the net inflammatory serum potential; thus, there is need for holistic assessments such as this endothelial biosensor method. Future work is required to address the value of this approach for prediction of risk, efficacy of therapies, or safety of pharmaceuticals. 


\section{Abbreviations}

BMI: Body mass index; CRRC: Cardiovascular risk reduction clinic; CAD: Coronary artery disease; CRP: C-reactive protein; HbA1C: Hemoglobin A1C; hCAECs: Human coronary artery endothelial cells; IL-8: Interleukin-8; ICAM-1: Intracellular adhesion molecule-1; MDA: Malondialdehyde; TNFa: Tumor necrosis factor-a; VCAM-1: Vascular cell adhesion molecule-1.

\section{Competing interests}

The authors declared that they have no competing interests.

\section{Authors' contributions}

$\mathrm{HC}$ optimized and conducted the endothelial cell bioassays, qPCR, and cytokine multiplex assays. MA was similarly involved in the conduct of technical aspects of the assays, trained HC on the cellular and molecular techniques. KZ contributed to the development of assays and analysis of data. JA and JN initiated the Cardiovascular Pharmacy Clinic, collected the blood samples along with much of the clinical chemistry and demographics data. AS collected/contributed the control patient serum samples. He also provided invaluable insight into the design and analysis of clinical data. CR consulted on the design and analyses and contributed to the writing. CQ conducted statistical analysis. MJC conceived the assay paradigm, funded, and provided overall stewardship of this innovative and collaborative project. All authors read and approved the final manuscript.

\section{Acknowledgements}

This project was supported in part by the National Center for Research Resources and the National Center for Advancing Translational Sciences (UL1 TR000041), National Institute of Environmental Health Sciences (ES014639) and National Institute of Occupational Safety and Health (OH010495). The content is solely the responsibility of the authors and does not necessarily represent the official views of the $\mathrm{NIH}$.

\section{Author details}

${ }^{1}$ Department of Pharmaceutical Sciences, College of Pharmacy, University of New Mexico, Albuquerque, NM, USA. ${ }^{2}$ Department of Pharmacy Practice and Administrative Sciences, College of Pharmacy, University of New Mexico, Albuquerque, NM, USA. ${ }^{3}$ Department of Internal Medicine, University of New Mexico, Albuquerque, NM, USA. ${ }^{4}$ Department of Biostatistics, School of Medicine, University of New Mexico, Albuquerque, NM, USA.

\section{Received: 22 December 2014 Accepted: 10 March 2015}

\section{Published online: 22 March 2015}

\section{References}

1. Ross R. Atherosclerosis-an inflammatory disease. N Engl J Med. 1999:340:115-26. doi:10.1056/NEJM199901143400207.

2. Corbi G, Bianco A, Turchiarelli V, Cellurale M, Fatica F, Daniele A, et al. Potential Mechanisms Linking Atherosclerosis and Increased Cardiovascular Risk in COPD: Focus On Sirtuins. Int J Mol Sci. 2013;14:12696-713. doi:10.3390/ijms140612696

3. Van Eeden S, Leipsic J, Paul Man SF, Sin DD. The relationship between lung inflammation and cardiovascular disease. Am J Respir Crit Care Med. 2012;186:11-6. doi:10.1164/rccm.201203-0455PP.

4. Lahousse L, van den Bouwhuijsen QJ, Loth DW, Joos GF, Hofman A, Witteman JC, et al. Chronic obstructive pulmonary disease and lipid core carotid artery plaques in the elderly: the Rotterdam Study. Am J Respir Crit Care Med. 2013;187:58-64. doi:10.1164/rccm.201206-10460C.

5. Kucukazman M, Ata N, Yavuz B, Dal K, Sen O, Deveci OS, et al. Evaluation of early atherosclerosis markers in patients with nonalcoholic fatty liver disease. Eur J Gastroenterol Hepatol. 2013;25:147-51. doi:10.1097/ MEG.0b013e32835a58b1.

6. Brea A, Mosquera D, Martin E, Arizti A, Cordero JL, Ros E. Nonalcoholic fatty liver disease is associated with carotid atherosclerosis: a case-control study. Arterioscler Thromb Vasc Biol. 2005;25:1045-50. doi:10.1161/01. ATV.0000160613.57985.18.

7. Barve S, Joshi-Barve S, Song Z, Hill D, Hote P, Deaciuc I, et al. Interactions of cytokines, S-Adenosylmethionine, and S-Adenosylhomocysteine in alcohol-induced liver disease and immune suppression. J Gastroenterol Hepatol. 2006;21 Suppl 3:S38-42. doi:10.1111/j.1440-1746.2006.04590.x.
8. McClain CJ, Hill DB, Song Z, Chawla R, Watson WH, Chen T, et al. S-Adenosylmethionine, cytokines, and alcoholic liver disease. Alcohol. 2002;27:185-92.

9. Liang YJ, Shyu KG, Wang BW, Lai LP. C-reactive protein activates the nuclear factor-kappaB pathway and induces vascular cell adhesion molecule-1 expression through CD32 in human umbilical vein endothelial cells and aortic endothelial cells. J Mol Cell Cardiol. 2006;40:412-20. doi:10.1016/j. yjmcc.2005.12.008.

10. Kawanami D, Maemura K, Takeda N, Harada T, Nojiri T, Saito T, et al. C-reactive protein induces VCAM-1 gene expression through NF-kappaB activation in vascular endothelial cells. Atherosclerosis. 2006;185:39-46. doi:10.1016/j.atherosclerosis.2005.01.057.

11. Montgomery KF, Osborn L, Hession C, Tizard R, Goff D, Vassallo C, et al. Activation of endothelial-leukocyte adhesion molecule 1 (ELAM-1) gene transcription. Proc Natl Acad Sci U S A. 1991;88:6523-7.

12. Yousuf $\mathrm{O}$, Mohanty BD, Martin SS, Joshi PH, Blaha MJ, Nasir K, et al. High-sensitivity C-reactive protein and cardiovascular disease: a resolute belief or an elusive link? J Am Coll Cardiol. 2013;62:397-408. doi:10.1016/j.jacc.2013.05.016.

13. Channell MM, Paffett ML, Devlin RB, Madden MC, Campen MJ. Circulating factors induce coronary endothelial cell activation following exposure to inhaled diesel exhaust and nitrogen dioxide in humans: evidence from a novel translational in vitro model. Toxicol Sci. 2012;127:179-86. doi:10.1093/ toxsci/kfs084.

14. Barua RS, Ambrose JA, Srivastava S, DeVoe MC, Eales-Reynolds LJ. Reactive oxygen species are involved in smoking-induced dysfunction of nitric oxide biosynthesis and upregulation of endothelial nitric oxide synthase: an in vitro demonstration in human coronary artery endothelial cells. Circulation. 2003;107:2342-7. doi:10.1161/01.CIR.0000066691.52789.BE.

15. Barbieri SS, Zacchi E, Amadio P, Gianellini S, Mussoni L, Weksler BB, et al. Cytokines present in smokers' serum interact with smoke components to enhance endothelial dysfunction. Cardiovasc Res. 2011;90:475-83. doi:10.1093/cvr/cvr032.

16. Agarwal B, Campen MJ, Channell MM, Wherry SJ, Varamini B, Davis JG, et al. Resveratrol for primary prevention of atherosclerosis: clinical trial evidence for improved gene expression in vascular endothelium. Int J Cardiol. 2013;166:246-8. doi:10.1016/j.ijcard.2012.09.027.

17. Robertson S, Colombo ES, Lucas SN, Hall PR, Febbraio M, Paffett ML, et al. CD36 mediates endothelial dysfunction downstream of circulating factors induced by $\mathrm{O}_{3}$ exposure. Toxicol Sci. 2013;134:304-11. doi:10.1093/toxsci/kft107.

18. Campen M, Robertson S, Lund A, Lucero J, McDonald J. Engine exhaust particulate and gas phase contributions to vascular toxicity. Inhal Toxicol. 2014;26:353-60. doi:10.3109/08958378.2014.897776.

19. Heyward VH. Practical body composition assessment for children, adults, and older adults. Int J Sport Nutr. 1998;8:285-307.

20. Emerging Risk Factors C, Kaptoge S, Di Angelantonio E, Pennells L, Wood AM, White IR, et al. C-reactive protein, fibrinogen, and cardiovascular disease prediction. N Engl J Med. 2012;367:1310-20. doi:10.1056/NEJMoa1107477.

21. Emerging Risk Factors C, Di Angelantonio E, Gao P, Pennells L, Kaptoge S, Caslake M, et al. Lipid-related markers and cardiovascular disease prediction. JAMA. 2012:307:2499-506. doi:10.1001/jama.2012.6571.

22. Sever PS, Poulter NR, Chang CL, Thom SA, Hughes AD, Welsh P, et al. Evaluation of C-reactive protein before and on-treatment as a predictor of benefit of atorvastatin: a cohort analysis from the Anglo-Scandinavian Cardiac Outcomes Trial lipid-lowering arm. J Am Coll Cardiol. 2013;62:717-29. doi:10.1016/j.jacc.2013.02.098.

23. Pearson T, Ballantyne C, Sisk C, Shah A, Veltri E, Maccubbin D. Comparison of effects of ezetimibe/simvastatin versus simvastatin versus atorvastatin in reducing C-reactive protein and low-density lipoprotein cholesterol levels. Am J Cardiol. 2007;99:1706-13. doi:10.1016/j.amjcard.2007.01.062.

24. Ikonomidis I, Andreotti F, Economou E, Stefanadis C, Toutouzas P, Nihoyannopoulos P. Increased proinflammatory cytokines in patients with chronic stable angina and their reduction by aspirin. Circulation. 1999;100:793-8.

25. Woodward M, Lowe GD, Francis LM, Rumley A, Cobbe SM, Investigators CS. A randomized comparison of the effects of aspirin and clopidogrel on thrombotic risk factors and C-reactive protein following myocardial infarction: the CADET trial. J Thromb Haemost. 2004;2:1934-40. doi:10.1111/j.1538-7836.2004.01017.x.

26. Haffner SM, Greenberg AS, Weston WM, Chen H, Williams K, Freed MI. Effect of rosiglitazone treatment on nontraditional markers of cardiovascular disease in patients with type 2 diabetes mellitus. Circulation. 2002;106:679-84. 
27. Sola S, Mir MQ, Lerakis S, Tandon N, Khan BV. Atorvastatin improves left ventricular systolic function and serum markers of inflammation in nonischemic heart failure. J Am Coll Cardiol. 2006;47:332-7. doi:10.1016/j.jacc.2005.06.088.

28. Solheim S, Pettersen AA, Arnesen $H$, Seljeflot I. No difference in the effects of clopidogrel and aspirin on inflammatory markers in patients with coronary heart disease. Thromb Haemost. 2006;96:660-4.

Submit your next manuscript to BioMed Central and take full advantage of:

- Convenient online submission

- Thorough peer review

- No space constraints or color figure charges

- Immediate publication on acceptance

- Inclusion in PubMed, CAS, Scopus and Google Scholar

- Research which is freely available for redistribution 